\title{
ATM Switch with GAMMA Network ; Provision of Multiple Paths
}

\author{
Keun-Bae Kim*, Sugwon Hong*, Kyu-Seob Cho** \\ and Byung-Chul Park** \\ * : Broadband Communications Department, \\ Electrical and Telecommunications Research Institute \\ Yosung P.O. BOX 106, Taejon 305-600, Korea \\ Phone : +82-42-860-5363 FAX : +82-42-861-5597 \\ ** : Department of Electronics Engineering, \\ Sung Kyun Kwan Univ. \\ 53, 3-Ka, Myungryun-Dong, Chongro-Ku, Seoul 110-745, Korea
}

\begin{abstract}
We propose an ATM switch architecture using the multistage interconnection network based on the GAMMA network. Consisting of $3 \times 3$ crossbar switch elements, the GAMMA network provides multiple paths. For this reason, this switch can be constructed without any sorting network which is required for other space division ATM switches based on the delta or banyan networks in order to avoid internal blocking. Most of the space division switches deteriorate switch performance when internal switch elements or interconnection links fail because they only one path between input and ouput ports. The proposed switch, however, has reliabilty by providing multiple paths with the GAMMA network.

This switch architecture has two network planes: the upper and lower plane. The upper plane is used for searching for the paths of the request cells, whearas the lower plane is used for routing the information cells. Since the switch can negotiate possible paths for next cell while one cell is routed through the lower plane, we can utilize the characteristics of the multiple paths in GAMMA network. Finally we show the performance of this switch by simulation.
\end{abstract}

\section{Keywords}




\section{INTRODUCTION}

Broadband ISDN based on the ATM technology is expected to provide a wide range of services from a few kbps of remote telemetry services to a few hundred Mbps of high-quality video transfer services. Since ATM was internationally agreed upon as a target switching mode, many types of ATM switch architectures have been proposed and nowadays many ATM switches are introduced in the market based upon a lot of different architectures. ATM swicth architectures which have been proposed so far can be classified as four categories: shared memory type, shared medium type, space division type, and fully interconnection type[1][7][3]. Among them, the space division type is the one to draw the attention of many researchers even though they have been implemented slowly compared to the other types.

Most space division switch architectures, using the multistage interconnection network(MIN) applied mainly in parallel processing architecture, are built on $2 \times 2$ crossbar type switches as a basic switch element, and are constructed by various interconnection network types such as Banyan, Flip, Omega, and the like[3][2]. Those kinds of interconnection networks, however, have only one path between input and output port, and usually require sorting networks to avoid internal blocking. The sorting networks itself need the basic switch elements of $(N / 4)\left\{\left(\log _{2} N\right)^{2}+\log _{2} N\right\}$ depending the number of input ports $\mathrm{N}$, subsequently making too many switch elements inside a switch. In addition, because of having only one path between input and output port, the interconnection networks mentioned above can not provide alternate paths if any switch element or link fails.

In order to cope with the drawbacks explained above and diversify the study of the space division type switches, we propose the architecture which is composed of the GAMMA network. The GAMMA network, consisting of $3 \times 3$ crossbar switch elements, can provide multiple paths[6][5]. The switch architecture has two network planes: the upper and lower planes. On the upper plane, the switch investigates whether any path is available to a destined output port. After it confirms that a path is available, it routes an information cell on the lower plane. Since two planes work in parallel, cell routing and path searching can be done simultaneously for different cells. That is, while one cell is being routed on the lower plane, the paths of next cells can be looked up using routing tags on the upper plane. Moreover, since the GAMMA network has multiple paths, this switch is more reliable even in the case of the failure of the switching elements or links.

The remaining of this paper is as follows. In the following section, we explain the property of the GAMMA network. In section 3, we describe the structure and operations of the switch, and we show the performance of this switch by simulation in section 4 . Finally, 
the conclusion is followed in section 5 .

\section{GAMMA AND IGAMMA NETWORK}

The GAMMA network consists of $\mathrm{S}$ stages, each stage having $\mathrm{N}$ switch elements, and each switch element is composed of 3 input links and 3 output links, providing the full $3 \times 3$ crossbar switching function [6]. The switch elements at each stage are interconnected by the PM2 (Plus-Minus $2^{\mathrm{I}}$ ) interconnection method[6][4]. The P-th switch element on the left of the $\mathrm{i}$-th stage is interconnected to the switch element on the right of the $\mathrm{i}$-th stage by the following relation:.

$$
\begin{aligned}
& \mathrm{PM} 2_{+i}(\mathrm{P})=\left(\mathrm{P}+2^{\mathrm{i}}\right), \text { modulo } \mathrm{N} \\
& \mathrm{PM} 2_{-\mathrm{i}}(\mathrm{P})=\left(\mathrm{P}-2^{\mathrm{i}}\right), \text { modulo } \mathrm{N} \\
& \mathrm{PM} 2_{\mathrm{S}}(\mathrm{P})=\mathrm{P}
\end{aligned}
$$

where $\mathrm{N}$ is the number of input ports, and $\mathrm{S}=\log _{2} \mathrm{~N}, 0 \leq \mathrm{i} \leq \mathrm{S}-1$.

Figure 1 shows an example of $N=8$. In this figure, the stages are ordered from 0 to $\mathrm{S}-1$, and the interconnections of switch elements at stage $i$ are made up of three links: $+2^{i}$ link(PM2 $\left.{ }_{+\mathrm{i}}\right),-2^{\mathrm{i}} \operatorname{link}\left(\mathrm{PM} 2_{-\mathrm{i}}\right)$, and a straight $\operatorname{link}\left(\mathrm{PM} 2_{\mathrm{S}}\right)$.

The paths in the GAMMA network are determined by the distance, $d$, which is defined as a destination port number minus a source port number. The multiple interconnection paths at each stage should satisfy the following relations:

$X_{i}=\left\{+2^{i},-2^{i}, 0\right\}$

$\delta=\sum_{i=0}^{S-1} X_{i}$ or

$\delta-N=\sum_{i=0}^{S-1} X_{i}$

where $\mathbf{X}_{\mathrm{i}}$ is a set has three element. In this way, more than 2 paths can exist if a source port number is different from a destination port number, and the numbers of available paths are varied depending on distances(see Table 1). In Figure 1, multiple paths are shown in bold lines when the source port number is 1 and the destination port number is 6 .

The inverse GAMMA(IGAMMA) network has the same structure, interconnection paths, and switch element functions as the GAMMA network except that IGAMMA is a 
mirror image of GAMMA network. An example of the IGAMMA network is shown in Figure 2.

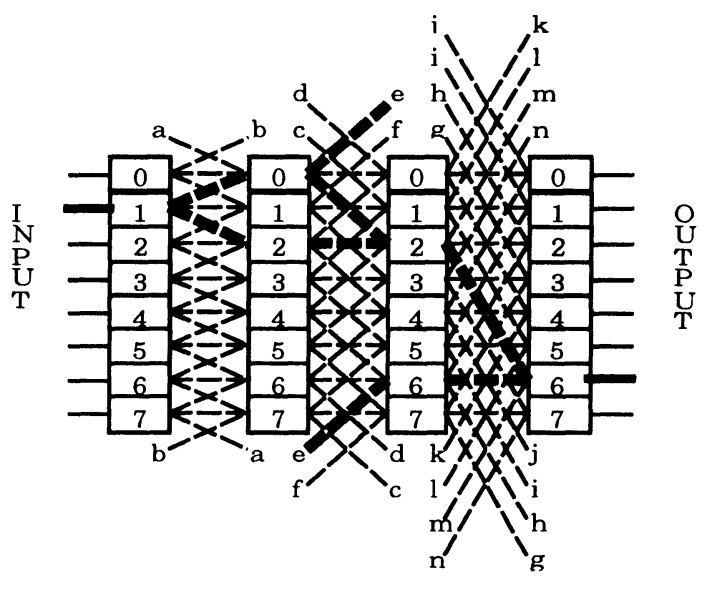

$\begin{array}{llll}\text { STAGE } & 0 & 1 & 2\end{array}$

Figure 1 The GAMMA network of $\mathrm{N}=8$

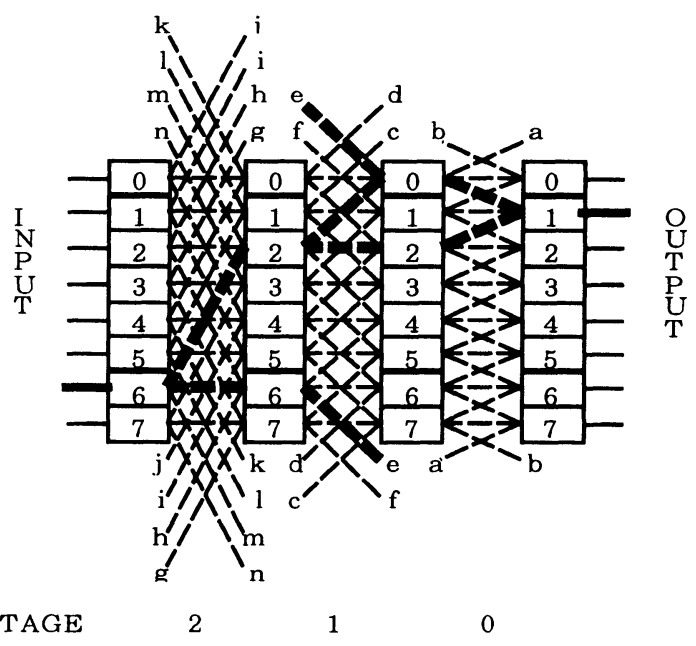

Figure 2 The Inverse GAMMA network of $\mathrm{N}=8$ 
Table 1 Number of paths according to the distance

\begin{tabular}{|c|c|c|c|c|c|c|}
\hline stance & 8 & 16 & 32 & 64 & 128 & 256 \\
\hline $\begin{array}{c}0 \\
1 \\
2 \\
3 \\
4 \\
5 \\
6 \\
7 \\
\vdots \\
\vdots 3 \\
14 \\
15 \\
\vdots \\
29 \\
30 \\
31 \\
\vdots \\
\vdots \\
61 \\
62 \\
63 \\
\vdots \\
\vdots \\
125 \\
126 \\
127 \\
\vdots \\
\vdots \\
253 \\
254 \\
256\end{array}$ & $\begin{array}{l}1 \\
4 \\
3 \\
5 \\
2 \\
5 \\
3 \\
4 \\
--\end{array}$ & $\begin{array}{l}1 \\
5 \\
4 \\
7 \\
3 \\
8 \\
5 \\
7 \\
\vdots \\
7 \\
7 \\
4 \\
5 \\
-\end{array}$ & $\begin{array}{r}1 \\
6 \\
5 \\
9 \\
4 \\
11 \\
7 \\
10 \\
\vdots \\
12 \\
7 \\
9 \\
\vdots \\
9 \\
5 \\
6 \\
--\end{array}$ & $\begin{array}{r}1 \\
7 \\
6 \\
11 \\
5 \\
14 \\
9 \\
13 \\
\vdots \\
17 \\
10 \\
13 \\
\vdots \\
16 \\
9 \\
11 \\
\vdots \\
\vdots \\
11 \\
6 \\
7 \\
--\end{array}$ & $\begin{array}{c}1 \\
8 \\
7 \\
13 \\
6 \\
17 \\
11 \\
16 \\
\vdots \\
22 \\
13 \\
17 \\
\vdots \\
23 \\
13 \\
10 \\
\vdots \\
\vdots \\
20 \\
11 \\
13 \\
\vdots \\
\vdots \\
13 \\
7 \\
8 \\
--\end{array}$ & $\begin{array}{c}1 \\
9 \\
8 \\
15 \\
7 \\
20 \\
13 \\
19 \\
\vdots \\
27 \\
16 \\
21 \\
\vdots \\
30 \\
17 \\
21 \\
\vdots \\
\vdots \\
29 \\
16 \\
19 \\
\vdots \\
\vdots \\
24 \\
13 \\
15 \\
\vdots \\
15 \\
8 \\
9\end{array}$ \\
\hline
\end{tabular}

\section{PROPOSED SWITCH STRUCTURE}

\subsection{Functional structure}

The switch architecture proposed in this paper based on the GAMMA network is shown in Figure 3. The switch structure consists of two planes: the upper plane and the lower plane. On the upper plane, available paths of incoming cells are examined using the routing tags. On the lower plane, information cells are transmitted if their paths are available on the upper plane. Three subnetworks constitute a switch network, and their functions are explained below. Figure 4 shows the actual structure of a $4 \times 4$ switch network. 


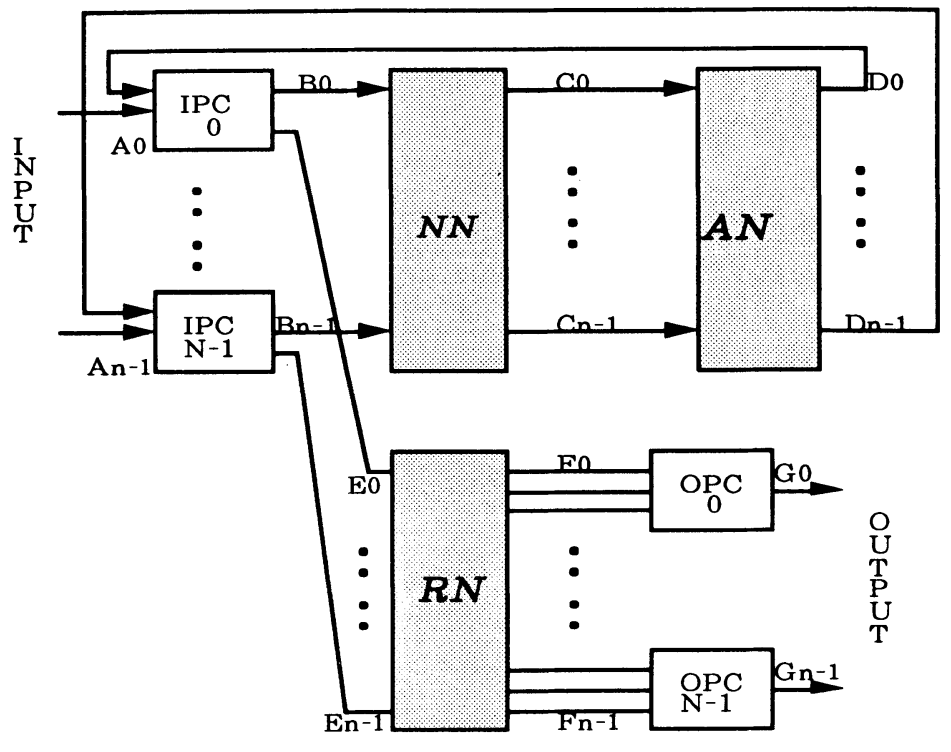

Figure 3 The Functional switch structure

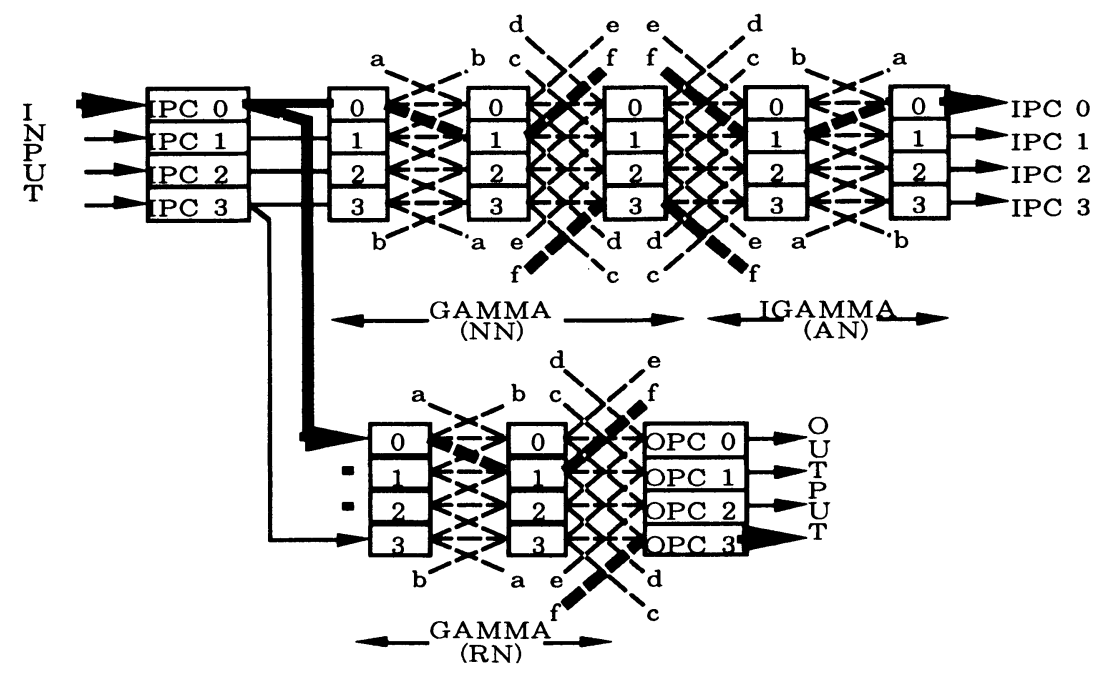

Figure 4 The $4 \times 4$ switch structure 


\section{o NN(Negotiation Network)}

NN searchs for possible paths using request cells including the routing tags. NN consists of a complete GAMMA network. But the switch elements of the final stage has three output links per switch element, and each output link is connected to each input link of AN(Acknowledge Network) which is located next to NN.

\section{o AN(Acknowledge Network)}

AN routes request cells which have passed through NN to the source input ports, informing them whether paths are available or not. AN consists of a modified IGAMMA network excluding the last stage (S-1), and subsequently the first input stage of AN has three input links coming from NN, and output links at the final stage are connected to IPCs(Input Port Controller)

\section{o RN(Routing Network)}

RN consists of a modified GAMMA network where the last stage (S-1) is eliminated. RN routes information cells from IPCs to OPCs(Output Port Controller), which have ACK signals from the upper plane. Since OPC is connected to each switching element at the final stage with three output links, OPC can receive up to maximum of three cells during one cell time.

The upper plane processes request cells having only routing tag, whereas the lower plane processes information cells with a full size including the routing tag. Thus, we have the relation between the processing time on the upper plane, $t$, and the processing time on the lower plane, $T$, such that $T \geq m t$ where $m$ is integer and $m \geq 1$. In other words, while RN transmits the (n-1)th cell, the upper plane can examine the path of the $\mathrm{n}$-th cell $m$ times. Using this feature, the switch can make the most use of the opportunity of multiple paths.

\subsection{Request cell and switch operation}

The format of the request cell which is used for examining paths is shown in Figure 5. The request cell consists of four kinds of bits: ACTivity bit, CONFirm bit, Sign bit and Magnitute bit. 


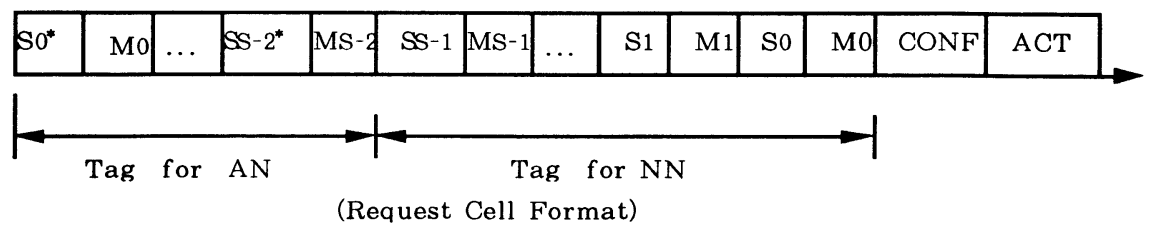

\begin{tabular}{|l|l|l|l|l|l|l|l|l|l|}
\hline Information & SS-1 & MS-1 & $\ldots$ & S1 & M1 & S0 & M0 & 1 & 0 \\
\hline
\end{tabular}

(Information Cell Format)

S : Sign bit $\begin{gathered}\text { M : Magnitude bit } \\ \text { ACT : ACTivity bit }\end{gathered}$

Figure 5 The format of the request cell and information cell

\section{o ACTivity bit}

This bit informs the switch elements at each stage that this incoming cell is valid. And it is also used for the recognition of ACK or NACK signal by IPC when IPC receives this cell from AN.

\section{- CONFirm bit}

If IPC receives ACK signal at the $i$-th request, then IPC modifies CONF bit to 1 in order to reserve the ACKed path during remaining $m-i$ searching periods. Where $m$ is a negotiation time which will be explained in next section..

\section{o Sign and Magnitude bit}

Sign and Magnitude bits are used for deciding output links at the switch elements at each stage. That is, if $M=1$ and $S=0$, then the $+2^{i}$ link is used, and if $M=1$ and $S=1$, then the $-2^{i}$ link is used, otherwise the straight link is used. NN uses $M_{0}$ to $S_{S-1}$ bits and $A N$ uses $M_{S-2}$ to $\mathrm{S}_{0}{ }^{*}$ bits for the routing tags. The order in $\mathrm{AN}$ is a reverse of the one in $\mathrm{NN}$ and the sign bits of AN are one's complement. In this way, the request cells which have passsd through NN can also pass through AN, consequently routing to the original source IPC.

Switch operation is performed through several phases such as start phase, negotiation phase, ACK phase, and data transfer phase. These phases on timing sequence are shown in Figure 6. 
In the start phase, the i-th IPC selects a cell at the head of its input buffer and obtains a distance $\delta$ which is defined as a destination address minus a source address. Using IPC retrieves $\mathrm{m}$ tag information from a routing data base and stores them in its own routing data queue(RDQ). And then IPC $_{i}$ reads the first tag from RDQ and makes a request cell by setting CONF and ACT bits to 0's. Finally the request cell is entered to NN through a $B_{i}$ link(see Figure 3).

In the negotiation phase, investigating paths depending on the routing tags of the request cells, NN sends the cells which have passed without conflicting with AN.

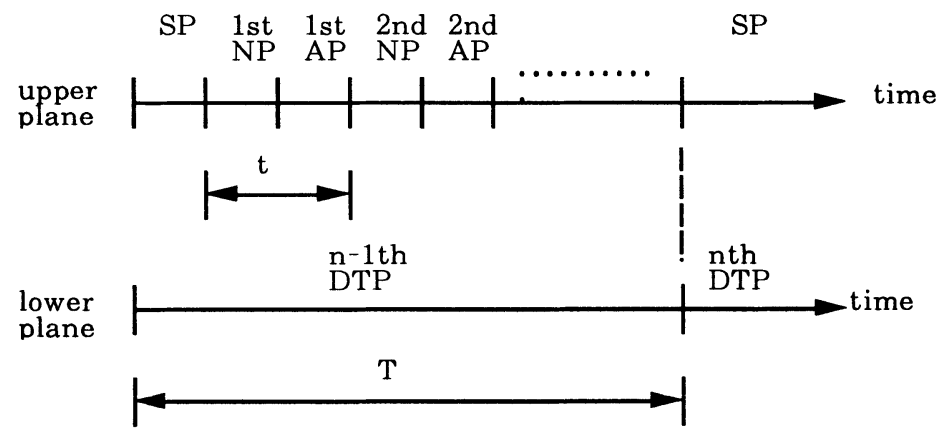

T >mt, SP : Start Phase, NP : Negotiation Phase AP : ACK Phase DTP': Data Transfer Phase

Figure 6 Switch operation on the time domain

In the ACK phase, AN delivers the confirmed request cells to the source IPCs, each IPC having a connection with each output port of $A N$. IPC $\mathrm{i}_{\mathrm{i}}$ carries out the following actions depending on the ACT bit information.

o bit=0: This cell is recognized as an ACK cell. It indicates that a path is available. The confirmed request cell with CONF bit set to 1 is formed to reserve the path at the next negotiation phase.

o bit=1: It notifies that there is no path on the request cell. In this case, $\operatorname{IPC}_{\mathrm{i}}$ retrieves new routing tag from RDQ and reconstructs a new request cell.

In this way, two kinds of request cells with the CONF bit set to 0 or 1 enter NN at the starting time of new negotiation phase. 
In the data transfer phase, finishing $m$ times of negotiation, IPCs which had ACK cells receive information cells and route them to destined OPCs through RN. IPCs which had no ACK cells retry transmission at the next time slot.

\subsection{Negotiation time and sorting method of routing tag}

\subsubsection{Negotiation time}

In this proposed switch structure, we can improve throughput if the switch has more negotiation opportunities for the $\mathrm{n}+1$ th cell while the $\mathrm{nth}$ cell is being routed through $\mathrm{RN}$. The negotiation opportunity is affected by negotiation time at NP(Negotiation Phase) and AP(ACK Phase) compared to the routing time of the information cells at RN. According to Figure 5 and 6, The maximun negotiation time is given by the ratio of RN processing time for each DTP(Data Transfer Phase) to negotiation and ACK processing time for each NP and AP. If we neglect processing time and propagation time at IPCs, and normalize processing time per bit at each switch element to 1 , then we have

$$
\begin{aligned}
& m=\frac{\text { transmission time of information cell }}{\text { processing time of request cell }}=\frac{(\text { tag size for } N N+\text { ACT \& CONF bit })+\text { info. cell size }}{\text { tag size for NN }+ \text { tag size for AN }+ \text { ACT \& CONF bit }} \\
& =\frac{(2 S+2)+(53 \times 8)}{2 S+2(S-1)+2}
\end{aligned}
$$

where $S=\log _{2} N$. According to equation (2), $N N$ can negotiate 27 times when $N=16$, and 13 times when $\mathrm{N}=256$.

\subsubsection{Routing tag sorting}

In the GAMMA network, the maximum number of paths $M$ varies depending on distance $\delta$, whereas the negotiation time $m$ in this switch is confined by the switch size. For this reason, it is necessary to choose $m$ paths among $M$ possible paths and store them in the routing data base. In figure 7, we show the possible routing paths of an example of $\mathrm{N}=64$ and distance $\delta=28$ (or -36 ). In this example, we have the maximum of 7 paths which is denoted by $1,-1$, and 0 for the $+2^{i}$ link, the $-2^{i}$ link, and the straight link respectively.

The ways to choosing $\boldsymbol{m}$ out of $M$ paths have many combinations and have relations with the priority assignment into the input links of the switching elements in the case of 
output conflict. Here we explain a priority assignment rule to be adopted in this paper.

o The cell with CONFbit=1 has the highest priority.

o If more than two cells with CONFbit $=0$ want to use the same output link, priorities are given by the input links which cells belong to, and their orders are the $+2^{i},-2^{i}$, and straight links.

Considering the above priority assignment rule, we consider the five sorting methods to choose $m$ out of $M$ based on the absolute values of the right hand side of equation (2) and select the method to have the best performance by simulation. The five sorting methods are described below and five cases of routing paths selected by those sorting methods for the example of $m=5$ are shown in Figure 8.

o method 1: The routing paths to have the smaller absolute values are selected first. If the absolute values are same, choose the path which has minus sign at the left most stage starting at $2^{\mathrm{S}-1}$. For example, in figure 7 , the second and third path has the same absolute value 36 . In that case the second path will be selected first because it has -1 at the $2^{5}$ stage, while the third path has +1 at the same stage.

o method 2: First, the routing paths to have larger absolute values are selected. If they are same, choose the path which has plus sign first in the same way as the method 1 except that it chooses one with plus sign.

o method 3: This mehod selects the paths which have the larger or the smaller absolute values alternatively one after andther starting from the largest. That is, first select the largest value, and then the smallest, and then the next largest, and then the next smallest, etc.

o method 4: This mehod selects the paths which have the smaller or the larger absolute values alternatively by turns starting from the smallest. This is the same way to select as the method 3 except starting from the smallest.

o method 5: This method chooses every other path starting from the largest absolute path.

There are many other sorting methods, and we need to find out which is the optimized sorting method. Here we leave it to futrher study. 


$\begin{array}{rrrrrrrlc}\text { stage }: & 2^{5} & 2^{4} & 2^{3} & 2^{2} & 2^{1} & 2^{0} & \text { disatnce }(\delta) & \text { absolute value } \\ 0 & 1 & 1 & 1 & 0 & 0 & 16+8+4=28 & 28 \\ -1 & 0 & 0 & -1 & 0 & 0 & -32-4=-36 & 36 \\ 1 & 0 & 0 & -1 & 0 & 0 & 32-4=28 & 36 \\ -1 & 0 & -1 & 1 & 0 & 0 & -32-8+4=-36 & 44 \\ 1 & 0 & -1 & 1 & 0 & 0 & 32-8+4=28 & 44 \\ -1 & -1 & 1 & 1 & 0 & 0 & -32-16+8+4=-36 & 60 \\ 1 & -1 & 1 & 1 & 0 & 0 & 32-16+8+4=28 & 60\end{array}$

Figure 7 Possible routing paths of the case of $N=64, \delta=28(-36)$

\begin{tabular}{rrrrrrrrrrrrrrrrrrr}
\multicolumn{11}{c}{ (case 1$)$} & \multicolumn{11}{c}{ (case 2$)$} \\
0 & 1 & 1 & 1 & 0 & 0 & 1 & -1 & 1 & 1 & 0 & 0 & 1 & -1 & 1 & 1 & 0 & 0 \\
-1 & 0 & 0 & -1 & 0 & 0 & -1 & -1 & 1 & 1 & 0 & 0 & 0 & 1 & 1 & 1 & 0 & 0 \\
1 & 0 & 0 & -1 & 0 & 0 & 1 & 0 & -1 & 1 & 0 & 0 & -1 & -1 & 1 & 1 & 0 & 0 \\
-1 & 0 & -1 & 1 & 0 & 0 & -1 & 0 & -1 & 1 & 0 & 0 & -1 & 0 & 0 & -1 & 0 & 0 \\
1 & 0 & -1 & 1 & 0 & 0 & 1 & 0 & 0 & -1 & 0 & 0 & 1 & 0 & -1 & 1 & 0 & 0
\end{tabular}

\begin{tabular}{rrrrrlrrrrrr}
\multicolumn{11}{c}{$($ case 4) } \\
0 & 1 & 1 & 1 & 0 & 0 & 1 & -1 & 1 & 1 & 0 & 0 \\
1 & -1 & 1 & 1 & 0 & 0 & 1 & 0 & -1 & 1 & 0 & 0 \\
-1 & 0 & -1 & 1 & 0 & 0 & -1 & 0 & 0 & -1 & 0 & 0 \\
-1 & -1 & 1 & 1 & 0 & 0 & 0 & 1 & 1 & 1 & 0 & 0 \\
1 & 0 & 0 & -1 & 0 & 0 & -1 & -1 & 1 & 1 & 0 & 0
\end{tabular}

Figure 8 Results of 5 sorting methods for figure 7, $m=5$

\section{PERFORMANCE EVALUATION}

We evaluate the performance of the switch, comparing throughputs depending on the various parameters such as switch size $(\mathrm{N})$, negotiation time $(m)$, and offered load $(\rho)$ by simulation. In this simulation, we assume that cells arrive at input ports uniformly with identical and independent distribution, and are routed to output ports with the same probabilities. This switch has input and output buffers. We assume that 3 outgoing cell streams are stored in the outpt buffers of OPC and then multiplexed into one output link(see Figure 3). The input buffer size is assumed to be infinite, and also the processing time at each module and the propagation time at each link are assumed to be zero. 
First, in order to evaluate the five sorting methods explained in the previous section, we obtain average throughput depending on those sorting methods by simulation, assuming the following conditions: $N=64, m=4$, and $\rho=0.7$. The results are shown in Figure 9. According to this figure, we can conclude that the method 3 has the best performance. Therefore, we use method 3 for the analysis of the switch from now on.

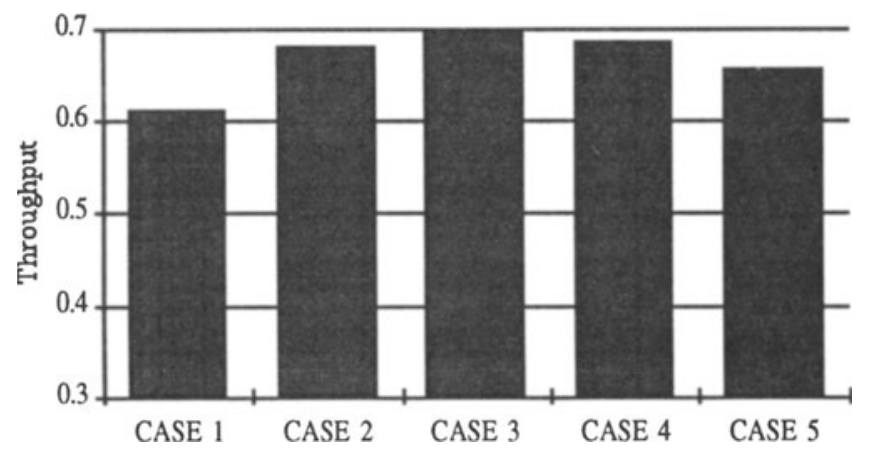

Figure 9 Throughput vs. sorting methods $(\mathrm{N}=64, m=4, \rho=0.7)$

Next, changing switch size, negotiation time, and offered load, we evaluate the performance of the switch. The results are shown in Figure 10 and Figure 11 . Figure 10 shows the maximum throughput under the variations of $m=1$ to $8, \rho=1$, and $N=8,16,32,64$, 128 , 256. At $m=8$, throughputs are $0.97,0.94,0.920 .88,0.83,0.78$ when $\mathrm{N}=8,16,32,64$, 128 , and 256 respectively. As negotiation time is increased, throughput is increased until $m$ $=5$. After $m=5$, however, the increase of thoughput is saturated. Since OPC can receive up to maximum of 3 cells, more than 5 times of negotiaion dose not affect throughput. But we have the excess searching time because the maximum negotiation time is greater than 5 on aveage, and this excess searching time can be used for reducing HOL(Head Of Line) blocking effect in the following way.

IPCs which have path assignments until $n(n<m)$ times of negotiation keep reserving the paths by setting CONFbit to 1 , and other IPCs find cells in the input buffer which have different destination address from cells used already during $n$ times of negotiation, and use these cells for path negotiation during $m-n$ times left.

Figure 11 shows throughput, varing $\rho=0.6$ to 1 . Incoming cells are transmitted without 
serious accumulation at input buffers until $\rho=0.7$, but cells will be delayed and lost seriously from $\rho=0.8$ except for $N=64$.

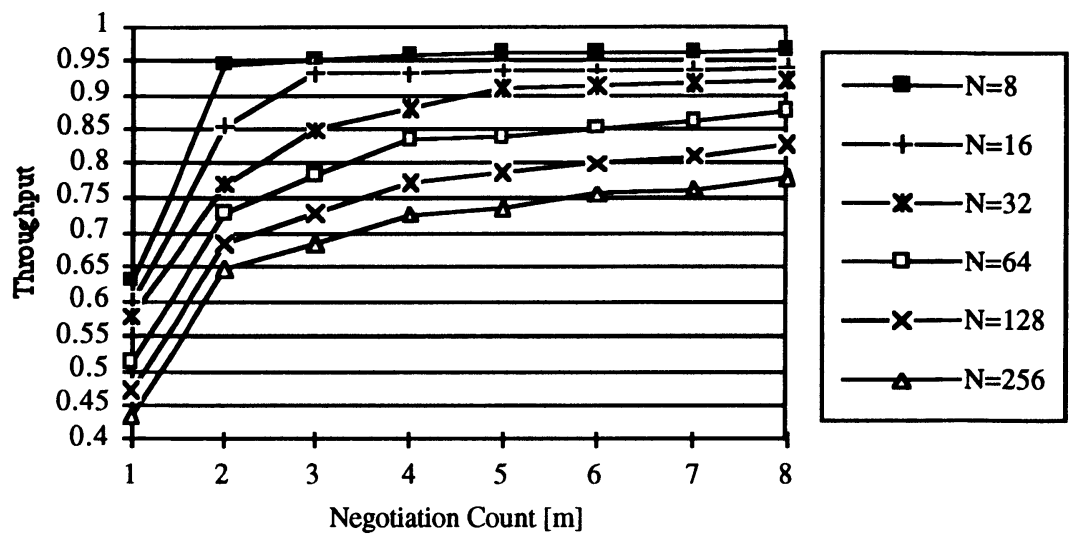

Figure 10 Throughput vs. negotiation time $(\rho=1)$

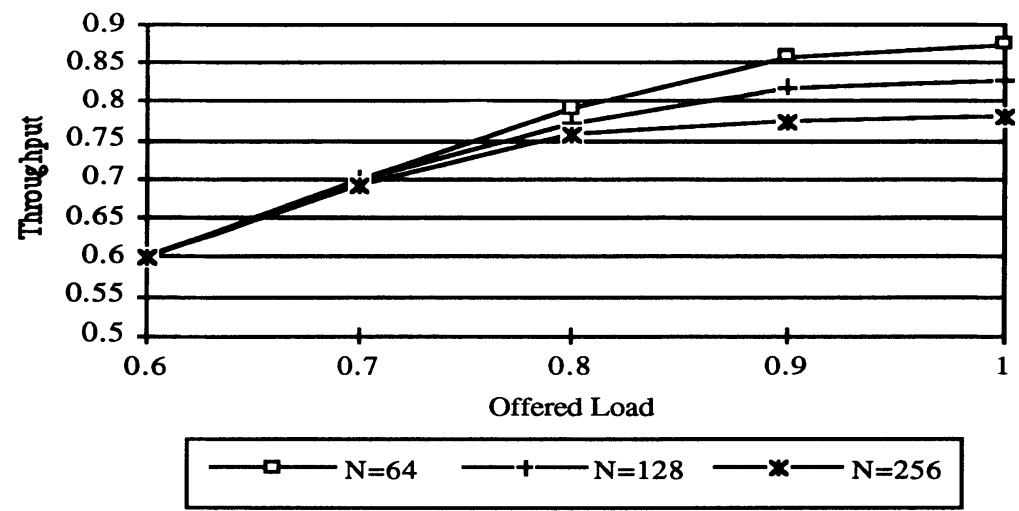

Figure 11 Throughput vs.offered $\operatorname{load}(m=8)$

\section{CONCLUSION}

In this paper, we propose the switch structure based on the GAMMA and IGAMMA networks which consist of $3 \times 3$ switch elements. This structure has several advantages over 
other space division switch architectures. First, providing multiple paths, it can prevent from degrading performance due to the failure of switch elements or links. It can also prevent cell loss in switch fabric by confirmimg paths before routing. The characteristics of multple paths also make the sorting network unnecessary which is required to other space division switches which used banyan or delta network..

As for throughput, this switch can process incoming traffic excellently until the switch size of $\mathrm{N}=64$ at $\rho=0.8$, whereas it needs overload control when it is applied to more than $\mathrm{N}=256$ and $\rho=0.7$. Finally VLSI technology is another important factor in introducing this kind of the switch structure. Contemporary switch architectures employ the $2 \times 2$ switch element as a basic unit. However, considering the development of VLSI and ASIC techonology, we can realize the ATM switches to use the $3 \times 3$ switch elements proposed in this paper. In this respect, we take aim at broadening the horizon of the ATM switch studies by proposing an ATM switch architecure with the GAMMA network which is different from other space division ATM switch architectures which are based on the Banyan types netwroks.

\section{REFERENCES}

[1] H. Ahmadi and W.E. Denzel, " A survey of modern high-performance switching techniques," IEEE J. SAC, vol.7, no7, Sep. 1989,pp.1091-1103.

[2] J. Giacopelli, M. Littlewood, and W.D. Sincoskie, " Sunshine: a high performance selfrouting broadband packet switch architecture," Int. Switching Symposium '90, Stockholm Sweden, May 27 - June 1, 1990, pp. 123-129.

[3] J. Y. Hui. "Switching and Traffic Theory for Integrated Broadband Networks," Kluwer Academic Publisher, 1990, pp.119-126.

[4] R. J. Mcmillen, " Routing schemes for the augmented data manipulator network in an MIMD system," IEEE Trans. on Computer, vol c-31, no 12, Dec. 1982, pp. 1202-1214.

[5] D.S. Parker, " The GAMMA network : multi-processor network redundants path," 9th Annual int'1 Symp. Computer Architecture, Apr. 1982

[6] H. J. Siegel, " Interconnection Networks for Large-Scale Parallel Processing," McGrowHill, 1989

[7] F. A. Tobagi, "Fast packet switch architectures for broadband integrated services networks, " proc. IEEE, Jan. 1990, pp.1133-1167 


\section{BIOGRAPHY}

Keun-Bae Kim received the B.E., M.E. and Ph.D. degrees in electronic engineering from Sung Kyun Kwan University, Korea, in 1984,1990 and 1993, respectively. From 1984 to 1988 he worked for Daewoo Telecom Research Center in Korea, where he worked on research and developmet of a digital exchange. In 1993 he worked at the Broadband Communications Department of Electronics and Telecommunication Research Institute, Taejon, Korea, where he has been engaged in research and development of a broaband access node system and an ATM switching System.

Sugwon Hong received the B.S. in physics from Seoul National University in 1979 and M.S. and Ph.D. in computer science from North Carolina University in 1988 and 1992 respectively. He worked at Korea Institute Science and Technology(KIST) and Korea Institue Energy and Resources(KIER) until 1986. Since 1993, he worked at the Broadband Communications department of Electronics and Telecommunications Research Institute(ETRI), where he has been working on the development of an ATM switching system and an ATM LAN system.

Kyu-Seob Cho received the B.E.in electronic engineering, M.E. in electric engineering, and $\mathrm{Ph} . \mathrm{D}$. degree in electronic engineering from Sung Kyun Kwan University, Seoul, Korea in 1974,1976 and 1989, respectively. From 1977 to 1992, he joined the Department of ISDN, Electronics and Telecommunications Research Institute, Taejon, Korea, where he was engaged in research and development on digital exchange, transmission networks, and ISDN. Since 1992, he hase been with the Department of Electronic Engineering, Sung Kyun Kwan University, Seoul, Korea, as an associate professor.

Byung-Chul Park received the B.E. in telecommunication engineering from Seoul National University. and Ph.D. degree in electric engineering from In Ha University, Incheon, Korea in 1957, 1975, respectively. From 1970 to 1972, he joined the Department of Electronic Engineering, Soon Sil University, Seoul, Korea, as an assistant professor. Since 1972, he joined the Department of Electronic Engineering, Sung Kyun Kwan University, Seoul, Korea, where he is currently professor. Dr. Park is currently an Supervisor of Korea Institute of Communication Sciences. 